assembling the data for analysis and contributed to writing the manuscript. HJ participated in the study design and revising the manuscript. JAK and $\mathrm{HJ}$ are guarantors for the paper.

Funding: No specific funding.

Competing interests: The Boston Collaborative Drug Surveillance Program is supported in part by grants from AstraZeneca, Berlex Laboratories, Boehringer Ingelheim Pharmaceuticals, Boots Healthcare International, Bristol-Myers Squibb Pharmaceutical Research Institute, GlaxoWellcome, Hoffmann-La Roche, Janssen Pharmaceutica Products, R W Johnson Pharmaceutical Research Institute; McNeil Consumer Products, and Novartis Farmaceutica. JAK is a John and Virginia Taplin fellow at the Harvard School of Public Health and is supported by a training fellowship in cancer epidemiology from the National Cancer Institute (T32-CA 09001).

1 Wakefield AJ, Murch SH, Anthony A, Linell J, Casson DM, Malik M, et al. Ileal-lymphoid-nodular hyperplasia, non-specific colitis, and pervasive developmental disorder in children. Lancet 1998;351:637-41.

2 Taylor B, Miller E, Farrington CP, Petropoulos M-C, Favot-Mayaud I, Li J, et al. Autism and measles, mumps, and rubella vaccine: no epidemiological evidence for a causal association. Lancet 1999;353:2026-9.

Wakefield AJ. MMR vaccination and autism. Lancet 1999:354:949-50.

4 Jick H. A database worth saving. Lancet 1997;350:1045-6.

4 Jick H. A database worth saving. Lancet 1997;350:1045-6. Jick H, Withers JM, Dean
Gen Pract 1995;45:107.

6 Wing L. The autistic spectrum. Lancet 1997;350:1761-6.

(Accepted 8 February 2000)

\title{
What parents are told after prenatal diagnosis of a sex chromosome abnormality: interview and questionnaire study
}

\author{
Lenore Abramsky, Sue Hall, Judith Levitan, Theresa M Marteau
}

\begin{abstract}
Objective To investigate how the prenatal diagnosis of a sex chromosome anomaly is first communicated to parents.

Design Health professionals were interviewed by telephone and the conversation was taped; parents were sent questionnaires at 1 month after diagnosis and those who responded were sent another at 6 months.

Participants 29 health professionals who had recently informed parents that a sex chromosome anomaly had been identified in an apparently anatomically normal, viable fetus. 23 mothers and partners who had been informed of such a diagnosis.

Main outcome measures Health professionals' knowledge about sex chromosome anomalies and parents' responses to information provided by health professionals.

Results Analysis of the telephone interviews identified great variation in what different healthcare professionals know, think, and say about the same sex chromosome anomaly. The small numbers and the low response rate for the questionnaire (39\% for women and 30\% for men) meant that statistical analysis was not appropriate.

Conclusions It is essential for obstetric units to have an established protocol for giving results and for all staff who communicate results to parents to have accurate, up to date information about the condition identified.
\end{abstract}

\section{Introduction}

Most fetuses and babies with an extra sex chromosome are not identified ${ }^{1}$ because there are usually no indications for karyotyping. ${ }^{2-6}$ However, a sex chromosome anomaly is sometimes detected prenatally when amniocentesis is performed to exclude Down's syndrome or other serious chromosomal anomalies. Conditions in which there is an extra sex chromosome are fundamentally different from those such as Down's syndrome in which affected individuals have recognisable characteristics that can be explained by laboratory findings. Prenatal detection of sex chromosome anomalies and other karyotypes or genotypes that may have no phenotypic consequences or only mild ones will become more common as testing becomes more widely available. ${ }^{7}$ Understanding the difficulties faced by health professionals in disclosing the prenatal diagnosis of a sex chromosome anomaly can highlight some of the problems that may be encountered during the prenatal detection of other atypical laboratory findings in fetuses with phenotypes that are likely to fall within the normal range (table 1).

We investigated how the prenatal diagnosis of a sex chromosome anomaly is first communicated to parents. We did not test a hypothesis but hoped to generate discussion about this issue. The first communication to parents is important because it may affect how information presented later is interpreted or even whether it is sought. ${ }^{8-10}$ Previous studies have looked at counselling that occurred once clinical geneticists
Editorial

by Biesecker

North Thames Perinatal Public Health Unit, Department of Medical and Community Genetics, Imperial College of Science, Technology, and Medicine,

Northwick Park

Site, Harrow

HA1 3UJ

Lenore Abramsky senior research officer

Judith Levitan research assistant

Psychology and Genetics Research Group, Guy's, King's College, and St Thomas's Hospitals School of Medicine, London SE1 9RT

Sue Hal

research associate

Theresa M Marteau professor

Correspondence to: L Abramsky

l.abramsky@ic.ac.uk

BMJ 2001;322:463-6

Table 1 Fetal karyotypes and characteristics ${ }^{2-6}$

\begin{tabular}{|c|c|c|c|c|c|}
\hline \multirow[b]{2}{*}{ Condition } & \multirow[b]{2}{*}{ Frequency } & \multicolumn{3}{|c|}{ Characteristics } & \multirow[b]{2}{*}{ Treatment } \\
\hline & & Intelligence quotient & Fertility & Other considerations & \\
\hline 47XXY (Klinefelter's syndrome) & 1 in 800 males & About $10-15$ points lower than siblings & Infertile & May have gynaecomastia & $\begin{array}{l}\text { Testosterone from } \\
\text { puberty }\end{array}$ \\
\hline 47XXX (Triple X) & 1 in 1000 females & About 20 points lower than siblings & Fertile & None & None \\
\hline 45X (Turner's syndrome)* & 1 in 2500 females & Unaffected & Infertile & $\begin{array}{l}\text { Short stature; may have } \\
\text { congenital malformations }\end{array}$ & $\begin{array}{l}\text { Oestrogen from puberty; } \\
\text { growth hormone }\end{array}$ \\
\hline
\end{tabular}

${ }^{\star}$ Turner's syndrome is common at conception but $98 \%$ of fetuses will be miscarried. 
Table 2 Fetal karyotype and parents' decisions about pregnancy

\begin{tabular}{lccccc} 
& No & $\begin{array}{c}\text { Mean (range) } \\
\text { maternal age } \\
\text { (years) at } \\
\text { Karyotype }\end{array}$ & & $\begin{array}{c}\text { Decision made about } \\
\text { pregnancy }\end{array}$ \\
\cline { 4 - 6 } & of cases & delimatery date & Continue & Terminate \\
\hline $47 X X Y$ (Klinefelter's syndrome) & 8 & $38.8(23-45)$ & 6 & 2 \\
\hline $47 X X Y / 46 X Y$ (Klinefelter's mosaic) & 1 & 37 & 1 & 0 \\
\hline $47 X Y Y$ & 3 & $35.3(34-37)$ & 2 & 1 \\
\hline $47 X Y Y / 45 X$ (mosaic) & 1 & 39 & 1 & 0 \\
\hline $45 X$ (Turner's syndrome) & 2 & $35.5(33-38)$ & 0 & 2 \\
\hline $47 X X X$ (Triple X syndrome) & 8 & $40.0(39-42)$ & 7 & 1 \\
\hline
\end{tabular}

became involved, ${ }^{11}$ but as one mother said during counselling after terminating a pregnancy affected by Klinefelter's syndrome: "I didn't really hear anything the geneticist said to me because I had already decided to have a termination."

\section{Participants and methods}

The study was done over 19 months (March 1998 to September 1999) in the North and South Thames Health Regions in England with approval from the local ethics committee and the multicentre regional ethics committee. Staff from cytogenetics laboratories in the two health regions notified us by telephone when they detected sex chromosome aneuploidy in an apparently anatomically normal, viable fetus. We telephoned the health professional who had made the initial disclosure of the diagnosis to the parents and conducted a brief, semi-structured telephone interview which was taped.

The interview took place within a few days of the disclosure and included questions about the manner in which the disclosure of the diagnosis had been made, the information that had been given, and the health professional's feelings and knowledge about the condition. If the initial disclosure involved little more than making an appointment for further discussion, we also interviewed the health professional who saw the parents later. The tapes were transcribed and reviewed by two of the authors (LA and SH). A total of 29 interviews about 23 cases were conducted with 16 midwives, 10 obstetricians, 2 genetic counsellors, and 1 general practitioner. The health professionals interviewed came from 14 district general hospitals and 3 fetal medicine units.

Laboratories failed to notify us of a few cases at the time of diagnosis, and these cases were not included in the study. Three doctors declined to participate. Cases in which fetal malformations were identified by ultrasound examination were not included in the study.

At one month after diagnosis and again at six months women and their partners were requested by their obstetric consultant to complete a brief postal questionnaire that asked for information about their general wellbeing, their feelings about the way they had been told of the diagnosis, and the decision that they had subsequently made about the pregnancy. Nine women and seven partners returned the first questionnaire; six of these women and four of their partners returned the second. The small numbers and the low response rate $(39 \%$ of women returned the first questionnaire and $30 \%$ of men) meant that statistical analysis was not appropriate, but the questionnaires provided interesting and revealing comments.

\section{Results}

Most of the 23 women in the study were in their 40 s, and most had undergone prenatal karyotyping because of their age. Seventeen of the 21 couples whose fetus had an extra sex chromosome decided to continue the pregnancy, but in the two cases in which non-mosaic Turner's syndrome was diagnosed the pregnancy was terminated (table 2).

Typically, a woman first learnt of the diagnosis when she received a telephone call at home from a midwife whom she had never met or had met only once. The initial conversation was usually less than 15 minutes long. This was followed within a few days by a consultation in person at the hospital, usually with an obstetrician. Nineteen of the 23 women (with or without their partners) saw a genetic specialist before making a decision about whether to continue the pregnancy. At least two thirds of the couples were given written information about the condition before they had any contact with the genetic service.

During the course of our study, one of the cytogenetic laboratories began faxing an information leaflet for patients to the health professional to whom they had reported the results. This allowed health professionals to familiarise themselves with the condition before speaking to parents and to have written information to offer parents. It was a relatively simple, inexpensive way to ensure that those who initially gave the result had access to accurate, up to date information. Health professionals who received this information said that they found it extremely helpful.

One health professional said: "I read the information from the fax which was very helpful, and I based my counselling around the very comprehensive information ... [I] basically prepared what I was going to say on the back of that."

Another said: "The thing that actually helped me quite a lot was having the sheet from [the cytogenetic lab] which I knew I could then actually give them ... [It] helped me enormously to feel that whilst I'm sure that there was something I didn't mention, at least there was a basis to then go on and consider the options further."

Our main finding was that there was enormous variation between different health professionals in what they knew, thought, and told parents about specific sex chromosome anomalies. Health professionals from 7 of the 14 district general hospitals said that it was a matter of chance that they had been the one to inform parents of the result.

\section{Klinefelter's syndrome}

\section{Health professionals}

Compare how these four health professionals told parents about a diagnosis of Klinefelter's syndrome.

One health professional who rated Klinefelter's syndrome as being of the same severity as Down's syndrome said that: "It wasn't Down syndrome but was another chromosome abnormality." When asked by the parents if the baby would be mentally retarded she told them: "It would be a bit, but I couldn't tell you how mentally retarded the child will be." She reported that the father said: "As long as it is a chromosome abnormality, we can now decide whether we want to carry on or not." The health professional who saw them the next day said that he "didn't want to reassure them as from 
what [he] could gather, they had made up their minds they were going to have a termination." The woman had a termination the next day without having seen a clinical geneticist.

A second health professional who had not been told the actual karyotype informed a couple that: "Their result had come back and it was abnormal and they had to go and see [the obstetric consultant] in the morning." According to the health professional, the mother became very tearful and the father became angry and abusive when his demands for more information met with no success. He asked the health professional: "Well what does that mean? Does it mean it's got three heads ... six legs?" The health professional said: "It was awful ... I felt quite shaken."

A third health professional who had to call parents to inform them that amniocentesis had identified Klinefelter's syndrome said: "I said, 'The news isn't very good,' and he said, 'I gathered that from the tone of your voice."”

A fourth health professional said: "I'd read up all I could before going in, and I read all the information from the fax [of the information leaflet from the genetic centre], which was very helpful. I based my counselling around the information, on the details they sent me."

\section{Parents}

Some comments made on the questionnaires by parents of boys with Klinefelter's syndrome make disturbing reading.

A father wrote: "The consultant appeared to know little about the condition but seemed to expect us to make a judgment based on the fact that an abnormal result had occurred. I am glad that we insisted on finding out more about the condition before we were willing to make our decision, otherwise we could have decided to terminate through ignorance aloneinstead we now have a lovely son."

A mother wrote: "In retrospect, I feel rather shocked that parents in our situation should have so routinely been offered the option of terminationparticularly without first being offered appropriate counselling ... [I] felt that research papers we were shown at the hospital were both outdated and one sided."

\section{XYY}

Health professionals

Those health professionals who had disclosed a $47 \mathrm{XYY}$ result had more up to date knowledge and all gave similar information to parents.

One reported telling parents: "I don't want you to worry about it, because it's not a major abnormality, but we do need to discuss it further, so can you come in today?... It's not something that is particularly likely to cause major abnormalities and there [isn't] any need for major concerns, but there are associations with it in terms of some behavioural disorders which obviously will vary very much with individuals."

\section{Triple X syndrome}

Health professionals

Consider the contrast between what these two health professionals said about 47XXX.
One health professional considered 47XXX to be "as devastating as Down syndrome" and said "that there was a possibility of mental retardation, intelligence down, stunted."

Another health professional who considered 47XXX to be a very mild condition told parents that: "She would be a perfectly normal baby and she would go to a school, a normal school, and she would grow up normally and that she had an extra chromosome... [I] explained to them that the child would look just like every other child and that she was a normal child and that her intelligence might not be quite as great as you would expect for her parents but that she would still cope in a normal school.'

\section{Parents}

Compare the comments of these parents of $47 \mathrm{XXX}$ girls.

A mother wrote: "The result of the amniocentesis was given over the telephone followed by some outdated information. I felt distraught when I first received the result-however the geneticist assured me that my baby would be perfectly normal, and she is! The initial information intimated that she could be severely retarded. Some people may have terminated at this stage without expert advice. This horrifies me."

A father wrote: "The discussions we had following the amnio result were very helpful in understanding the condition. We have no regrets whatsoever concerning the pregnancy." The mother of the same girl said: "Our daughter is absolutely delightful; words cannot express how much joy she brings me!"

\section{Discussion}

It is disturbing to note the haphazard nature of how parents were informed of the diagnosis, what information was given, and what was implied. Some maternity units in the study reported that they had a set protocol for giving results whereas in others the reporting seemed to be done on an ad hoc basis. Many health professionals said that it was a matter of chance that they had been the one to inform parents of the results. What and how parents were told depended to a large extent on where they had their pregnancy care

\section{Suggestions for improving practice}

- All units should have a protocol for giving results; it should follow professional guidelines on the screening, diagnosis, and management of fetal abnormalities $^{18}$

- Women undergoing prenatal karyotyping should be informed of the existence of sex chromosome anomalies and of other variations from the norm before undergoing tests

- Up to date information about the clinical implications of the diagnosis, prepared by clinical geneticists, should be provided by the cytogenetic laboratory that makes the diagnosis along with the results of the test

- All staff who are likely to speak to parents about the results of diagnostic tests, including midwives who telephone parents to invite them to meet with a doctor, should be familiar with the diagnosis and read the information provided 


\section{What is already known on this topic}

Sex chromosome anomalies as a group are as common as Down's syndrome, but most affected individuals are never identified

Affected fetuses are sometimes identified when women have prenatal karyotyping for Down's syndrome

The diagnosis is almost always first disclosed to parents by staff from the obstetric unit, because there has not previously been an indication for a clinical genetics referral

\section{What this study adds}

Some obstetric units have no established protocol for communicating results to parents

Some health professionals working in an obstetric setting know little about the effect of sex chromosome anomalies

Some parents are given misleading information when they are first informed that their fetus has a sex chromosome anomaly

and who informed them of the results. Although there were some examples of excellent counselling, there were other examples of grossly inadequate or frankly misleading information being given. We can only speculate about how this variation might affect parents as our study was not designed to determine associations between the quality of counselling and the outcome of the pregnancy or subsequent emotional wellbeing of the parents. ${ }^{12-17}$ Some units providing prenatal testing services are not adhering to the published guidelines concerning the provision of information (box). ${ }^{18}$

We would like to thank the cytogeneticists from all the laboratories in the North and South Thames Health Regions for notifying us of appropriate cases. We also thank all the doctors, midwives, and counsellors who agreed to be interviewed; the parents who answered our questionnaires; Elizabeth Winchester and Liz Redfern for their assistance; and Christine Garrett and Sue Holder for their helpful comments. We also thank Hazel Showell for transcribing the tapes. This study was conducted as part of the European Union concerted action from Biomed 2 programme.

Contributors: LA formulated the original idea for the study and took a primary role in designing and executing it, interpret- ing the results, writing the paper, and will act as guarantor. $\mathrm{SH}$ helped design the study, interpret the results, and write the paper. JL helped design the study and interpret the results. TMM helped design the study, interpret the results, and write the paper.

Funding: TMM is funded by the Wellcome Trust. LA received an NHS Executive research and development grant.

Competing interests: None declared.

1 Abramsky L, Chapple J. 47XXY (Klinefelter syndrome) and 47XYY: estimated rates and indication for postnatal diagnosis with implications for prenatal counselling. Prenat Diagn 1997:17:363-8

2 Pennington BF, Bender B, Puck M, Salbenblatt J, Robinson A. Learning disabilities in children with sex chromosome anomalies. Child Dev 1982;53:1182-92.

3 Ratcliffe SG, Butler GE, Jones M. Edinburgh study of growth and development of children with sex chromosome abnormalities. In: Ratcliffe SG, Paul N, eds. Sex chromosome aneuploidy: prospective studies on children. New York: Alan R Liss, 1991:1-44. (Birth defects: original article series.)

4 Robinson A, Bender B, Linden M. Prognosis of prenatally diagnosed children with sex chromosome aneuploidy. Am J Med Genet 1992;44:365-8.

5 Garrett C, Carlton L. Difficult decisions in prenatal diagnosis. In: Abramsky L, Chapple J, eds. Prenatal diagnosis: the human side. London: Chapman and Hall, 1994:86-105.

6 Gotz MJ, Johnstone EC, Ratcliffe SG. Criminality and antisocial behaviour in unselected men with sex chromosome abnormalities. Psychol Med 1999;29:953-62.

7 Bentley DR. The human genome project-an overview. Med Res Rev 2000;20:189-96.

8 Tversky A, Kahneman D. The framing of decisions and the psychology of choice. Science 1981;211:453-8.

9 Marteau T. Framing of information: its influence upon decisions of doctors and patients. Br J Soc Psychol 1989;28:89-94.

10 Anderson G. Nondirectiveness in prenatal genetics: patients read between the lines. Nurs Ethics 1999;6:126-36.

11 Clayton-Smith J, Andrews T, Donnai D. Genetic counselling and parental decisions following antenatal diagnosis of sex chromosome aneuploidies. J Obstet Gynaecol 1989;10:5-7.

12 Puck MH. Some considerations bearing on the doctrine of self-fulfilling prophecy in sex chromosome aneuploidy. Am J Med Genet 1981;9:129-37.

13 Reif M, Baitsch H. Psychological issues in genetic counselling. Hum Genet 1985;70:193-9.

14 Robinson A, Bender BG, Linden MG. Decisions following the intrauterine diagnosis of sex chromosome aneuploidy. Am J Med Genet 1989;34:552-4.

15 Edwards-Beckett J. Child development: what do parents expect? Child Care Health Dev 1992:18:355-63.

16 Meschede D, Louwen F, Nippert I, Holzgreve W, Miny P, Horst J. Low rates of pregnancy termination for prenatally diagnosed sex chromosome polysomies. Am J Med Genet 1998;480:330-4.

17 Christian SM, Koehn D, Pillay R, MacDougall A, Wilson RD. Parental decisions following prenatal diagnosis of sex chromosome aneuploidy: a trend over time. Prenat Diagn 2000;20:37-40.

18 Joint Working Party of the Royal College of Obstetricians and Gynaecologists and the Roval College of Paediatrics and Child Health. Fetal abnormalities-guidelines for screening, diagnosis and management: report of a joint working party of the Royal College of Obstetricians and Gynaecologists and the Royal College of Paediatrics and Child Health. London: RCOG, 1997.

(Accepted 9 November 2000)
Centre for Public

Health Monitoring,

London School of

Hygiene and

Tropical Medicine,

London

WC1E 7HT

S Harding

senior epidemiologist

R Balarajan

professor

continued over

BMJ 2001;322:466-7
We previously reported high mortality and high incidence of cancer in second generation Irish people (children of Irish migrants) living in England and Wales. ${ }^{12}$ In this study we examine the mortality of third generation Irish people (grandchildren of Irish migrants) living in England and Wales.

\section{Method and results}

The longitudinal study by the Office for National Statistics is a record linkage study of a $1 \%$ representa- tive sample of the population of England and Wales. The sample was first extracted from the 1971 census and is updated with new births and immigrants. Information from censuses and registrations of vital events is linked to the records of study members. ${ }^{3}$

First generation Irish classified by country of birth covered those people born in Northern Ireland and the Irish Republic. For second and third generations, only those people with parents and grandparents born in the Republic of Ireland could be identified because parents born in Northern Ireland 